\title{
Psychotropic medication treatment patterns in community-dwelling schizophrenia in China: comparisons between rural and urban areas
}

\author{
Cai-Lan Hou ${ }^{1 \dagger}$, Shi-Bin Wang ${ }^{1 \dagger}$, Fei Wang ${ }^{1}$, Ming-Zhi Xu ${ }^{1}$, Miao-Yang Chen², Mei-Ying Cai ${ }^{3}$, Yao-Nan Xiao ${ }^{2}$ and \\ Fu-Jun Jia ${ }^{1 *}$
}

\begin{abstract}
Background: To date no study has compared more specifically the psychotropic medication treatment patterns for patients with schizophrenia living in community between rural and urban areas. This study examined the ruralurban differences of the use of psychotropic drugs among community-dwelling individuals with schizophrenia in China.

Method: Data on 993 community-dwelling patients with schizophrenia ( $n=479$ in rural area and $n=514$ urban area) were collected by interviews during 2013-2014, and 2015-2016 according to the diagnosis of DSM-IV or ICD-10. Data on patients' socio-demographic and clinical characteristics, prescriptions of psychotropic drugs were collected using a standardized protocol and data acquisition procedure.

Results: Multivariate analyses revealed that in comparison with the rural counterparts, the patients from the urban area were significantly more frequently prescribed antipsychotic polypharmacy, clozapine, and benzodiazepines, but the patients from the rural area had more frequently prescribed anticholinergics.

Conclusions: Substantial variations in psychotropic medication treatment patterns for patients with schizophrenia living in community were found between rural and urban areas in China. Common use of antipsychotic polypharmacy, clozapine and benzodiazepines in urban area, and anticholinergics in rural area need to be further addressed.
\end{abstract}

Keywords: Community-dwelling, Schizophrenia, Prescription, Rural, Urban

\section{Background}

There exist significant variations in the socio-economic status, culture, demographics and history of urban and rural places which influence mental health, while access to appropriate health services and health professionals are important determinants of treatment of schizophrenia.

Most of the studies on urban-rural differences in patients with schizophrenia focused on their epidemiological and aetiological factors [1-3], but urban-rural differences in psychotropic drug treatment model for

\footnotetext{
* Correspondence: jiafujun@126.com

${ }^{\dagger}$ Cai-Lan Hou and Shi-Bin Wang contributed equally to this work.

${ }^{1}$ Guangdong Mental Health Center, Guangdong General Hospital \& Guangdong Academy of Medical Sciences, 7/F, No.123, Huifuxi Road, Guangzhou, Guangdong Province, China

Full list of author information is available at the end of the article
}

people with schizophrenia living in the community are not well studied.

A number of factors associated with the availability, organization, and delivery of mental health services in rural and urban communities could also explain the differences in psychotropic use [3]. Rural communities may encounter place-specific challenges to population health, including geographic isolation, or stagnant economic growth in some areas [4]. Especially in China, general practitioners (GPs) have no permission of prescribing anti-psychotic medications except for benzodiazepines. Fewer psychiatrists in rural area than in urban area may also affect the medication treatment pattern.

It is attractive to explore differences in psychotropic medication treatment patterns and investigate related

(c) The Author(s). 2019 Open Access This article is distributed under the terms of the Creative Commons Attribution 4.0 International License (http://creativecommons.org/licenses/by/4.0/), which permits unrestricted use, distribution, and 
factors that may be the basis for any differences observed. Therefore, in the present study, we addressed two questions including what was the medication treatments currently used in community settings in urban and rural areas respectively and what factors were associated with choice of medication strategy in urban and rural area?

\section{Methods}

\section{Research design and subjects}

This study was an extension of a cross-sectional pharmaco-epidemiological survey on prescription trends of psychotropic medications [5] conducted between August, 2013 and March 2016 in Guang Zhou and Luo Ding, Guangdong province, China. Guang Zhou is the capital city of Guangdong province that is an urban area, while Luo Ding, a typical rural area lies in western Guangdong province and is underdeveloped.

Patients who met the following criteria were consecutively recruited: (1) aged equal to or greater than 18 years; (2) being diagnosed as schizophrenia according to DSM-IV or ICD-10; (3) being treated by primary care physicians; (4) having ability to understand the content of the interview and (5) being able to provide written informed consent. If the patients were antipsychotic-free, they would be excluded from the study. The study protocol was approved by the Ethics Committees of Guangdong Mental Health Centre (ethical code is Z2013-002). All patients provided written informed consent.

In China, most of the community-dwelling patients with schizophrenia who have presented to hospitals and primary care services are registered in the Chinese $\mathrm{Na}$ tional Psychiatric Management System. The recruitment processes of this cross-sectional study were as follows: 22 primary care service centers in Guangzhou (urban area) and 21 primary care service centers in Luo Ding (rural area) were selected randomly. Primary care physicians in the selected service centers contacted the randomly selected patients by telephone to provide a detailed introduction about this study procedure. Six training psychiatrists conducted interviews with the patients. The initial sample size is 1200 , and 600 patients in urban and rural areas, respectively.

\section{Assessments}

Basic socio-demographic and clinical data including prescription of psychotropic drugs were collected by a review of their electronic medical records. We only recorded and analyzed the present prescribing condition (active medications). Doses of antipsychotic drugs were converted into the prescribed daily dose/the defined daily dose ratio (PDD/DDD ratio) [5-7].
This study focused on the following major groups or aspects of treatments viz. second generation antipsychotics (SGAs) except clozapine, antipsychotic polypharmacy, clozapine, first generation antipsychotics (FGAs), antipsychotics free, benzodiazepines, mood stabilizers, antidepressants and anticholinergics. Clozapine was singled out due to its special place in the use of treatmentresistant patients. Antipsychotic polypharmacy (APP) was defined as co-prescription of 2 or more antipsychotics $[5,8]$.

The Brief Psychiatric Rating Scale (BPRS) was administered to assess psychotic symptoms $[9,10]$. Adverse effects of antipsychotics were assessed using the Simpson and Angus Scale of Extrapyramidal Symptoms (SAS) [11]. The 10-item Montgomery-Asberg Scale (MADRS) was used to measure depressive symptoms $[12,13]$.

\section{Statistical analysis}

SPSS 20.0 for Windows was used to analyze the data. The comparisons between rural and urban patients with regard to basic socio-demographic and clinical characteristics were performed using independent sample ttest, U test, and chi-square test, as appropriate. Multiple logistic regression analyses with the "Enter" method was conducted to examine the independent relationships between areas and prescribing patterns. For antipsychotic polypharmacy, participants were classified as APP and not APP categories. SGA and FGA were also classified as binary variables. Any use of SGAs except clozapine, antipsychotic polypharmacy, clozapine, FGAs, antipsychotic free, benzodiazepine, mood stabilizers, antidepressants and anticholinergics were entered as the dependent variables separately, while rural/urban area was an independent variable; demographic variables and clinical characteristics that showed significant differences between the two groups in aforementioned univariate analyses were adjusted for as covariates. The two-tailed significance level was set at 0.05 .

\section{Results}

In the end, a total of 993 patients with schizophrenia (514 in urban and 479 in rural areas) were available and included in the analyses. The response rate was $85.67 \%$ in urban areas and $79.83 \%$ in rural areas, respectively.

Socio-demographic and clinical data of the whole sample and also separately by urban and rural areas were showed in Table 1 . There were significant differences between the two groups in terms of gender, marital status, first episode, personal income, coverage of health insurance, family history of psychiatric disorders, major medical conditions, use of clozapine, SGAs (except clozapine), APP, antidepressants, benzodiazepines and anticholonergics, age, education level, illness duration, BMI, severity of depressive, negative and anxiety 
Table 1 Socio-demographic and clinical characteristics and psychotropic drug prescription in urban and rural areas ${ }^{a}$

\begin{tabular}{|c|c|c|c|c|c|c|c|c|c|}
\hline & \multicolumn{2}{|c|}{$\begin{array}{l}\text { Total } \\
(N=993)\end{array}$} & \multicolumn{2}{|c|}{$\begin{array}{l}\text { Urban } \\
(N=514)\end{array}$} & \multicolumn{2}{|c|}{$\begin{array}{l}\text { Rural } \\
(N=479)\end{array}$} & \multirow[t]{2}{*}{$x^{2}$} & \multirow[t]{2}{*}{$d f$} & \multirow[t]{2}{*}{$P$} \\
\hline & $n$ & $\%$ & $\mathrm{n}$ & $\%$ & $n$ & $\%$ & & & \\
\hline Male gender & 586 & 59.0 & 273 & 53.1 & 313 & 65.3 & 15.3 & 1 & $<0.001$ \\
\hline Married & 393 & 39.6 & 182 & 35.4 & 211 & 44.1 & 7.7 & 1 & 0.005 \\
\hline Employed & 661 & 66.6 & 342 & 66.5 & 319 & 66.6 & 0 & 1 & 0.98 \\
\hline First episode & 219 & 22.1 & 163 & 31.7 & 56 & 11.7 & 57.8 & 1 & $<0.001$ \\
\hline Personal income over 3000 yuan & 24 & 2.4 & 19 & 3.7 & 5 & 1.0 & 7.3 & 1 & 0.007 \\
\hline No health insurance & 126 & 12.7 & 122 & 23.7 & 4 & 0.8 & 117.3 & 1 & $<0.001$ \\
\hline Psychiatric family history & 195 & 19.6 & 137 & 26.7 & 58 & 12.1 & 25.6 & 1 & $<0.001$ \\
\hline Major medical conditions & 231 & 23.3 & 201 & 39.1 & 30 & 6.3 & 149.8 & 1 & $<0.001$ \\
\hline Clozapine & 357 & 36.0 & 222 & 43.2 & 135 & 28.2 & 24.2 & 1 & $<0.001$ \\
\hline FGA & 431 & 43.4 & 231 & 44.9 & 200 & 41.8 & 1.02 & 1 & 0.31 \\
\hline SGA except Clozapine & 411 & 41.4 & 161 & 31.3 & 250 & 52.2 & 44.5 & 1 & $<0.001$ \\
\hline APP & 310 & 31.2 & 193 & 37.5 & 117 & 24.4 & 19.8 & 1 & $<0.001$ \\
\hline Antidepressants & 40 & 4.0 & 28 & 5.4 & 12 & 2.5 & 5.5 & 1 & 0.01 \\
\hline Mood stabilizers & 189 & 19.0 & 108 & 21.0 & 81 & 16.9 & 2.7 & 1 & 0.1 \\
\hline Benzodiazepine & 161 & 16.2 & 128 & 24.9 & 33 & 6.9 & 59.2 & 1 & $<0.001$ \\
\hline \multirow[t]{2}{*}{ Anticholinergics } & 432 & 43.5 & 187 & 36.4 & 245 & 51.1 & 21.9 & 1 & $<0.001$ \\
\hline & Mean & SD & Mean & SD & Mean & SD & $t / u$ & $d f$ & $P / Z$ \\
\hline Age,y & 43.2 & 11.6 & 46.8 & 10.2 & 39.4 & 11.8 & 10.6 & 991 & $<0.001$ \\
\hline Education,y & 9.4 & 2.7 & 10.5 & 2.8 & 8.3 & 2.2 & 13.3 & 991 & $<0.001$ \\
\hline Age of onset,y & 25.6 & 9.1 & 25.1 & 8.9 & 26.0 & 9.3 & -1.6 & 991 & 0.103 \\
\hline Illness duration,y & 17.6 & 11.1 & 21.7 & 10.3 & 13.3 & 10.1 & 12.8 & 991 & $<0.001$ \\
\hline No. Of admissions & 2.2 & 2.4 & 2.4 & 2.7 & 2.1 & 2.03 & -1.1 & $\ldots$ & $0.26^{b}$ \\
\hline $\mathrm{BMI}, \mathrm{kg} / \mathrm{m}^{2}$ & 23.6 & 4.1 & 24.7 & 4.8 & 22.5 & 2.4 & 8.7 & 985 & $<0.001$ \\
\hline PDD/DDD & 0.9 & 1.2 & 0.9 & 1.6 & 0.8 & 0.5 & 1.7 & 991 & 0.08 \\
\hline MADRS total score & 7.4 & 8.2 & 10.01 & 9.3 & 4.7 & 5.7 & 10.6 & 991 & $<0.001$ \\
\hline \multicolumn{10}{|l|}{ BPRS score } \\
\hline positive & 5.9 & 2.8 & 6.1 & 3.1 & 5.8 & 2.4 & 1.9 & 991 & 0.053 \\
\hline negative & 5.7 & 2.8 & 6.2 & 3.08 & 5.3 & 2.5 & 4.8 & 991 & $<0.001$ \\
\hline anxiety & 4.2 & 2.03 & 3.2 & 1.7 & 5.2 & 1.8 & -18.2 & 991 & $<0.001$ \\
\hline SAS total score & 11.7 & 4.2 & 5.2 & 0.2 & 2.2 & 0.1 & 9.8 & 991 & $<0.001$ \\
\hline
\end{tabular}

Abbreviations: APP antipsychotic polypharmacy, BMI body mass index, BPRS brief psychiatric rating scale, FGA first-generation antipsychotic, MADRS MontgomeryAsberg Depression Rating Scale, PDD/DDD prescribed daily dose/the defined daily dose ratio, SAS Simpson and Angus scale of extrapyramidal symptoms, SGA second-generation antipsychotic

a Boldface indicates a statistically significant difference $(P<0.05)$

b Mann-Whitney $U$ test was used

symptoms, and extrapyramidal symptom. Multiple logistic regression analyses revealed that after controlling for covariates, compared to rural patients, urban patients were more likely to receive APP, clozapine, and benzodiazepines, but had less frequently prescribed anticholinergics (Table 2).

\section{Discussion}

To the best of our knowledge, this was the first study that examined the urban-rural differences of psychotropic medication treatment patterns in Chinese community-dwelling patients with schizophrenia. We found that rural patients had less use of antipsychotic polypharmacy, clozapine, and benzodiazepine, and more use of anticholinergics by contrast with urban patients. This cross-sectional study of prescribing patterns in the real-world settings might reflect the practice and treatment patterns due to several reasons.

The possible reason for discrepancies in prevalence rates of antipsychotic polypharmacy among studies 
Table 2 The independent associations of psychotropic drug prescription between rural/urban area

\begin{tabular}{|c|c|c|c|}
\hline & $P$ & Odds ratio & 95\% C.I. \\
\hline \multicolumn{4}{|c|}{ SGA, except clozapine ${ }^{a}$} \\
\hline Rural & 0.92 & 1.02 & $0.67-1.55$ \\
\hline Urban & - & 1 & - \\
\hline \multicolumn{4}{|c|}{ Antipsychotic polypharmacy-APP a } \\
\hline Rural & 0.002 & 0.5 & $0.32-0.77$ \\
\hline Urban & - & 1 & - \\
\hline \multicolumn{4}{|c|}{ Clozapine $^{\text {a }}$} \\
\hline Rural & 0.003 & 0.52 & $0.34-0.81$ \\
\hline Urban & - & 1 & - \\
\hline \multicolumn{4}{|l|}{$\mathrm{FGA}^{\mathrm{a}}$} \\
\hline Rural & 0.08 & 0.71 & $0.47-1.05$ \\
\hline Urban & - & 1 & - \\
\hline \multicolumn{4}{|c|}{ Benzodiazepines ${ }^{a}$} \\
\hline Rural & $<0.001$ & 0.28 & $0.16-0.51$ \\
\hline Urban & - & 1 & - \\
\hline \multicolumn{4}{|c|}{ Mood stabilizer ${ }^{a}$} \\
\hline Rural & 0.32 & 0.76 & $0.44-1.31$ \\
\hline Urban & - & 1 & - \\
\hline \multicolumn{4}{|c|}{ Antidepressants ${ }^{a}$} \\
\hline Rural & 0.15 & 0.44 & $0.14-1.34$ \\
\hline Urban & - & 1 & - \\
\hline \multicolumn{4}{|c|}{ Anticholinergics ${ }^{a}$} \\
\hline Rural & 0.04 & 1.503 & $1.01-2.24$ \\
\hline Urban & - & 1 & - \\
\hline
\end{tabular}

Abbreviations: APP antipsychotic polypharmacy, FGA first-generation antipsychotic, SGA second-generation antipsychotic

${ }^{a}$ : gender, marital status, first episode, personal income, coverage of health insurance, family history of psychiatric disorders, major medical conditions, age, education level, illness duration, BMI, and severity of depressive, negative and anxiety symptoms, as well as the extrapyramidal symptoms were controlled for as covariates

could be explained by differences in socio-demographic characteristics of the patients with schizophrenia, such as age, gender, educational level, income, and family support. A recent study reported that the place of residence was found to be remarkably related to polypharmacy and urban outpatients with schizophrenia had more APP by bivariate analysis, however multivariate logistic regression did not find the significant meaning [14]. Our finding on APP was similar to the previous study. The levels of antipsychotic polypharmacy were typical with community settings [15]. Rural communities have distinct socioeconomic and cultural characteristics from urban communities. Psychiatrists with lower education levels in rural areas may prefer monotherapy, while their urban counterparts may pursue a higher control rate and better continuous education could encourage them for polypharmacy [16]. On the other hand, patients with schizophrenia in urban areas with higher education could demand more health resources and accept higher spending on the antipsychotic for a higher quality of life [5]. In addition, relatively difficult access to psychological services and mental health professionals in rural places were perhaps influencing factors.

We also found that the SAS scores were significantly higher in urban group than in rural group, which could also be a result of the more antipsychotic polypharmacy in urban areas. However, the patients with schizophrenia from the urban areas had less frequently use of anticholinergics, which could relieve the iatrogenic extrapyramidal symptoms (EPS) of antipsychotic drugs. And there is little evidence supporting improved efficacy of APP, but it could increase adverse effect, mortality, cost of treatment and reduce treatment adherence. Additionally, the shortage of medical resources in rural areas may lead the rural psychiatric hospitals store less type and quantity of antipsychotics, rural clinical settings are more likely to have typical antipsychotics/FGAs, which could cause EPS. Besides reducing the side effects of antipsychotics, anticholinergic could also be used for COPD, asthma and Parkinson's disease. Data showed the prevalence rates of related chronic diseases in rural areas were higher than those in urban areas [26]. So the general practitioners (GPs) in rural areas may prescribe the relatively cheap anticholinergic for comorbidities of patients with schizophrenia [19]. This merits more research and concerns by the psychiatrists and professionals.

About $30 \%$ of individuals with schizophrenia have treatment-resistant conditions, and clozapine is the only antipsychotic with known efficacy in treatmentrefractory schizophrenia [17]. Previous survey in the United States found that treatment resistance and living in a rural area with historically high rates of clozapine prescription were the powerful predictors of clozapine prescription [18]. We found in this study that rural patients were prescribed less than urban patients although the price of clozapine is cheap and common use in China [19]. Although clozapine has a distinctive role in the treatment of schizophrenia because of its unique advantage, psychiatrists should pay more attention to its specific risks especially including agranulocytosis, metabolic syndrome, myocarditis and bowel obstruction [20]. Furthermore, the lack of medical human resource in rural areas could not meet the monitoring requirements for clozapine [21]. The potential risks, the mandatory blood monitoring as well as the insufficient knowledge on treatment of the risks may decrease the prescription of clozapine in rural areas. The US study reported that the geographic variation and that local practice model significantly influenced clozapine use [18]. However, there were also controversial studies showing that 
patients treated at university hospitals mainly located in the more urban areas were less likely to prescribed clozapine compared to individuals treated at nonuniversity hospitals mainly located in less urban areas. This also reflected the region difference on drug prescription [22, 23].

Rural patients were prescribed less benzodiazepine in this study, showing the rural doctors perhaps were less likely to prescribe the benzodiazepines for schizophrenic patients, which was contrary to some studies. In a study of tranquillizer prescribing in two municipal and two rustic areas of the United Kingdom, Gabe and Williams found that rustic doctors were more likely to prescribe tranquilizers as their practice expanded compared to urban doctors [24]. Cutts and Tett found similar trends in their study of prescribing patterns in Queensland, Australia [25].

There were several strengths of the present study including the large, homogeneous and randomly selected sample, as well as using the standard conversion method of antipsychotic dosage. However, the results should be interpreted with cautiously due to several limitations of methodology. First, the causality between different variables was difficult to get because of this study's cross-sectional survey. Second, only patients with schizophrenia during clinically stable phase living in communities were enrolled from one metropolitan city and one rural area. Therefore, the conclusions may not be universally applied to different parts of China and to other subject cohorts due to the limitation of representativeness. Third, there was no exact data on the proportion of the Chinese National Psychiatric Management System registered schizophrenic patients in the total patients with schizophrenia. Selection bias indeed existed due to the missing of the patients who did not access to the institutes. And due to unequal response rate between rural and urban areas (85.67\% in urban and $79.83 \%$ in rural), selection bias may also exist in the study. Despite these limitations, our data have health policy implications. The marked use of APP, clozapine and benzodiazepine in urban areas in our study, and the marked use of anticholinergics in rural areas in our study may be warranted by evidence of better efficacy and relapse prevention and reduced side effects.

In conclusion, substantial variations in psychotropic medication treatment patterns for community-dwelling schizophrenia were found between rural and urban area in China. Common use of antipsychotic polypharmacy, clozapine, and benzodiazepine in urban areas and anticholinergic use in rural areas need to be further addressed. Our findings suggested that further research would be needed to investigate the determining factors for these differences in psychotropic use.

\section{Acknowledgements}

The authors thank all the clinicians for their contribution to this study.

\section{Authors' contributions}

CLH and FJJ conceived and designed the study. SBW, FW, MYC and YNX collected data from participants. CLH and SBW analyzed, interpreted the data and prepared the manuscript. FJJ and MZX supervised and contributed to analysis and interpretation of the results of the manuscript. All the authors reviewed and approved the final manuscript.

\section{Funding}

The study was supported by Guangdong Provincial Department of Science and Technology (grant number: 2016A020215192; 2018A030313816), Guangdong Provincial Administration of traditional Chinese Medicine (grant number: 20171007) and Special Fund for Science and Technology of Guangdong Provincial People's Hospital (grant number: 2017zh02).

\section{Availability of data and materials}

All data generated and analysed during this study are included in this published article. Other data acquisition could contact the correspondents.

\section{Ethics approval and consent to participate}

The whole study was reviewed and approved by the Ethics Committee of Guangdong Mental Health Center, and the Ethical code is Z2013-002. Written informed consent was obtained from each participant.

\section{Consent for publication}

Not applicable.

\section{Competing interests}

The author Fujun Jia is currently acting as an Associate Editor for BMC Psychiatry. Other authors declare that they have no competing interests.

\section{Author details}

'Guangdong Mental Health Center, Guangdong General Hospital \& Guangdong Academy of Medical Sciences, 7/F, No.123, Huifuxi Road, Guangzhou, Guangdong Province, China. ${ }^{2}$ The Third People's Hospital of Luoding, Luoding, Guangdong Province, China. ${ }^{3}$ Guangzhou Yuexiu Center for Disease Control and Prevention, Guangzhou, Guangdong Province, China.

Received: 23 August 2018 Accepted: 18 July 2019

Published online: 05 August 2019

\section{References}

1. Pedersen CB, Mortensen PB. Are the cause(s) responsible for urban-rural differences in schizophrenia risk rooted in families or in individuals? Am J Epidemiol. 2006;163(11):971-8.

2. Pedersen $C B$, Mortensen PB. Why factors rooted in the family may solely explain the urban-rural differences in schizophrenia risk estimates. Epidemiol Psichiatr Soc. 2006;15(4):247-51.

3. van Os J, et al. Prevalence of psychotic disorder and community level of psychotic symptoms: an urban-rural comparison. Arch Gen Psychiatry. 2001; 58(7):663-8.

4. Hart LG, Larson EH, Lishner DM. Rural definitions for health policy and research. Am J Public Health. 2005;95(7):1149-55.

5. Hou CL, et al. Antipsychotic polypharmacy and quality of life in patients with schizophrenia treated in primary care in China. Int I Clin Pharmacol Ther. 2016:54(1):36-42.

6. $\mathrm{WHO}, \mathrm{WHO}$ Collaborating Center for Drug Statistics Methodolgy. 2011.

7. Bauer $M$, et al. Drug treatment patterns in bipolar disorder: analysis of longterm self-reported data. Int J Bipolar Disord. 2013;1:5.

8. Roh D, et al. Antipsychotic prescribing patterns in first-episode schizophrenia: a five-year comparison. Clin Psychopharmacol Neurosci. 2015; 13(3):275-82.

9. Overall JE, Beller SA. The brief psychiatric rating scale (BPRS) in geropsychiatric research: I. factor structure on an inpatient unit. J Gerontol. 1984:39(2):187-93.

10. Zhang MY, Zhou T, Tang SH, Chi YF, Xia ML, Wang ZY. The application of the Chinese version of the brief psychiatric rating scale (BPRS) (in Chinese) Chin J Nerv Ment Dis. 1983;9:76-80. 
11. Simpson GM, Angus JW. A rating scale for extrapyramidal side effects. Acta Psychiatr Scand Suppl. 1970;212:11-9.

12. Zhong BL, Wang Y, Chen HH, Wang XH. Reliability, validity and sensitivity of Montgomery-Åsberg depression rating scale for patients with current major depressive disorder (in Chinese). Chin J Behav Med Brain Sci. 2011;20:85-7.

13. Montgomery SA, Asberg M. A new depression scale designed to be sensitive to change. Br J Psychiatry. 1979;134:382-9.

14. Tesfaye $\mathrm{S}$, et al. Prevalence of antipsychotic polypharmacy and associated factors among outpatients with schizophrenia attending Amanuel mental specialized hospital, Addis Ababa, Ethiopia. Psychiatry J. 2016;2016:6191074.

15. STAHL S. Essential psychopharmacology. Neuroscientific basis and practical applications. 2nd ed. Cambridge: Cambridge University Press; 2000.

16. Beard JR, et al. Influence of socioeconomic and cultural factors on rural health. Aust J Rural Health. 2009;17(1):10-5.

17. Kane J, et al. Clozapine for the treatment-resistant schizophrenic. A doubleblind comparison with chlorpromazine. Arch Gen Psychiatry. 1988;45(9): 789-96.

18. Stroup TS, et al. Geographic and clinical variation in clozapine use in the United States. Psychiatr Serv. 2014;65(2):186-92.

19. Hou CL, et al. Clozapine prescription and quality of life in Chinese patients with schizophrenia treated in primary care. Pharmacopsychiatry. 2015;48(6): 200-4.

20. Henderson DC, et al. Clozapine, diabetes mellitus, hyperlipidemia, and cardiovascular risks and mortality: results of a 10-year naturalistic study. J Clin Psychiatry. 2005;66(9):1116-21.

21. Chinese Medical Association., C.M. Guideline for the prevention and treatment of psychiatric disorders in China, vol. 2003. Beijing: Chinese Medical Association; 2003.

22. Nielsen J, et al. Geographical and temporal variations in clozapine prescription for schizophrenia. Eur Neuropsychopharmacol. 2012;22(11): 818-24.

23. Wimberley $T$, et al. Inverse association between urbanicity and treatment resistance in schizophrenia. Schizophr Res. 2016;174(1-3):150-5.

24. Gabe J, Williams P. Rural tranquility?: urban-rural differences in tranquilliser prescribing. Soc Sci Med. 1986;22(10):1059-66

25. Cutts C, Tett SE. Influences on doctors' prescribing: is geographical remoteness a factor? Aust J Rural Health. 2003:11(3):124-30

26. Wang $\mathrm{S}$, et al. Rural-urban differences in the prevalence of chronic disease in Northeast China. Asia Pac J Public Health. 2015;27(4):394-406.

\section{Publisher's Note}

Springer Nature remains neutral with regard to jurisdictional claims in published maps and institutional affiliations.

Ready to submit your research? Choose BMC and benefit from:

- fast, convenient online submission

- thorough peer review by experienced researchers in your field

- rapid publication on acceptance

- support for research data, including large and complex data types

- gold Open Access which fosters wider collaboration and increased citations

- maximum visibility for your research: over $100 \mathrm{M}$ website views per year

At $\mathrm{BMC}$, research is always in progress.

Learn more biomedcentral.com/submissions 